\title{
Hayabusa Re-entry: Trajectory Analysis and Observation Mission Design
}

\author{
Alan M. Cassell ${ }^{1}$, Michael W. Winter ${ }^{2}$, Gary A. Allen, $\mathrm{Jr}^{3}$, Jay H. Grinstead ${ }^{4}$ \\ NASA Ames Research Center, Moffett Field, CA 94035-1000 \\ Manny E. Antimisiaris ${ }^{5}$ \\ NASA Dryden Flight Research Center, PO Box 273, Edwards, CA 93523-0273 \\ James Albers ${ }^{6}$, Peter Jenniskens ${ }^{7}$ \\ SETI Institute, 189 Bernardo Avenue, Mountain View, CA 94043
}

On June $13^{\text {th }}$, 2010, the Hayabusa sample return capsule successfully re-entered Earth's atmosphere over the Woomera Prohibited Area in southern Australia in its quest to return fragments from the asteroid 1998 SF36 "Itokawa". The sample return capsule entered at a super-orbital velocity of $12.04 \mathrm{~km} / \mathrm{sec}$ (inertial), making it the second fastest human-made object to traverse the atmosphere. The NASA DC-8 airborne observatory was utilized as an instrument platform to record the luminous portion of the sample return capsule re-entry $(\sim 60$ sec) with a variety of on-board spectroscopic imaging instruments. The predicted sample return capsule's entry state information at $\sim 200 \mathrm{~km}$ altitude was propagated through the atmosphere to generate aerothermodynamic and trajectory data used for initial observation flight path design and planning. The DC8 flight path was designed by considering safety, optimal sample return capsule viewing geometry and aircraft capabilities in concert with key aerothermodynamic events along the predicted trajectory. Subsequent entry state vector updates provided by the Deep Space Network team at NASA's Jet Propulsion Laboratory were analyzed after the planned trajectory correction maneuvers to further refine the DC-8 observation flight path. Primary and alternate observation flight paths were generated during the mission planning phase which required coordination with Australian authorities for pre-mission approval. The final observation flight path was chosen based upon trade-offs between optimal viewing requirements, ground based observer locations (to facilitate post-flight trajectory reconstruction), predicted weather in the Woomera Prohibited Area and constraints imposed by flight path filing deadlines. To facilitate sample return capsule tracking by the instrument operators, a series of two racetrack flight path patterns were performed prior to the observation leg so the instruments could be pointed towards the region in the star background where the sample return capsule was expected to become visible. An overview of the design methodologies and trade-offs used in the Hayabusa re-entry observation campaign are presented.

\section{Nomenclature}

$\begin{array}{ll}\text { ARC } & =\text { Ames Research Center } \\ \text { ATV } & =\text { Automated Transfer Vehicle } \\ \text { DFRC } & =\text { Dryden Flight Research Center } \\ \text { DSN } & =\text { Deep Space Network } \\ \text { EDL } & =\text { Entry, Descent and Landing } \\ \text { EFPA } & =\text { Entry Flight Path Angle } \\ \text { JAXA } & =\text { Japan Aerospace Exploration Agency } \\ \text { JPL } & =\text { Jet Propulsion Laboratory } \\ \text { OD } & =\text { Orbital Dynamics }\end{array}$

\footnotetext{
${ }^{1}$ Systems Engineer, ERC Inc., Entry Systems and Vehicle Development Branch, M/S 229-1, Associate Member.

${ }^{2}$ Research Scientist, UARC-University of California-Santa Cruz, Aerothermodynamics Branch, M/S 230-4, Associate Member.

${ }^{3}$ Aerospace Engineer, ERC Inc., Aerothermodynamics Branch, M/S 230-4, non-member.

${ }^{4}$ Hayabusa Observation Mission Project Manager, Aerothermodynamics Branch, M/S 230-4, Associate Fellow.

${ }^{5}$ Systems Engineer, Volunteer, Associate Member.

${ }^{6}$ Hayabusa Observation Mission Principal Investigator, Associate Fellow.
} 
SRC $=$ Sample Return Capsule

$\mathrm{TCM}=$ Trajectory Correction Maneuver

TLE $=$ Two Line Element

TPS $=$ Thermal Protection System

WPA $=$ Woomera Prohibited Area

\section{Introduction}

$\mathrm{O}$ June 13, 2010, the Hayabusa spacecraft completed its tumultuous seven-year mission when it delivered a sample return capsule (SRC) containing particles from the asteroid Itokawa onto the southern Australian Outback. ${ }^{1}$ The mission had its share of anomalies, including propulsion system fuel leak, communications black-out and ionengine malfunctions which threatened to jeopardize the mission. Nonetheless, the Hayabusa mission team was able to overcome these challenges and successfully navigated the spacecraft towards the Woomera Prohibited Area (WPA) in Southern Australia. After a successful entry, descent and landing (EDL) in the WPA, a recovery team retrieved the sample canister. The sample canister was returned to Japan for examination at the curation facility after which JAXA announced that particles found in the Hayabusa SRC came from the asteroid Itokawa, becoming the first mission to return a sample from a near Earth object. ${ }^{2}$

Hayabusa's return presented an opportunity to observe the re-entry of a capsule at superorbital velocities. Since the heatshield did not contain instrumentation, remote observation was the only option for acquiring data during reentry. The primary goal of the observation was to characterize the aerothermodynamic environment of re-entry with time-resolved measurements of optical emission from the SRC's surface, shock-heated gases ahead of the SRC, and the trailing wake. NASA's DC-8 airborne laboratory is ideally suited for optical imaging of re-entry events. The DC-8's cruise altitude of $\sim 12 \mathrm{~km}$ is above most clouds and atmospheric moisture which can absorb optical emission from the spacecraft. Because the full range of aerothermodynamic environments cannot be replicated in a single ground test, data from actual flight events, like Hayabusa's SRC re-entry, can serve as benchmarks for validation of end-to-end simulation tools used for the design and analysis of atmospheric entry systems. The entry velocity of Hayabusa's SRC was the second fastest into Earth's atmosphere. Building upon previous re-entry observation missions (Genesis, Stardust and ATV) ${ }^{3-5}$, the Hayabusa Observation Mission team successfully executed the airborne observation campaign. ${ }^{6}$ This paper presents an overview of the calculated Hayabusa SRC trajectory and methods utilized to design the observation flight track. The mission planning tools utilized key event timing and location information as inputs for designing observation flight tracks that would meet the mission objectives. The parameters influencing the observation mission, the analysis techniques for trading the various observation flight path options and the utilization of instrument pointing methods are described.

\begin{tabular}{|l|l|}
\hline \multicolumn{1}{|c|}{ Objectives/Constraints } & \multicolumn{1}{c|}{ Rationale } \\
\hline $\begin{array}{l}\text { Maintain velocity vector to observer } \\
\text { angle of }<45 \mathrm{deg} \text { through peak heating }\end{array}$ & $\begin{array}{l}\text { Maximize the amount of the front surface } \\
\text { that is visible }\end{array}$ \\
\hline $\begin{array}{l}\text { Maintain }<16 \mathrm{deg} \text { elevation angle during } \\
\text { peak heating window }\end{array}$ & Ease of tracking through DC-8 windows \\
\hline$<250 \mathrm{~km}$ range to SRC at peak heating & Ensures enough brightness \\
\hline $\begin{array}{l}\text { Maintain }+/-60 \mathrm{~km} \text { keep out zone on } \\
\text { either side of ground track }\end{array}$ & Safety- Debris avoidance \\
\hline
\end{tabular}

\section{Re-entry Observation Mission Overview}

During the weeks prior to re-entry, several trajectory correction maneuvers (TCMs) were executed to target Hayabusa's SRC EDL onto the WPA. ${ }^{1}$ During this time preparations were underway to install the instruments on board the NASA DC-8. Since there were some uncertainties concerning the successful execution of the TCMs, 
contingency plans and decision analyses were formulated to facilitate stakeholder assessments in case an off nominal event were to impact the planned observation mission. Figure 1 shows a timeline of terminal approach key events, observation mission key decision points, and orbital dynamic solution updates. The critical WPA targeting maneuver (TCM-3) was successfully executed by the $5^{\text {th }}$ of June, 2010. Once this critical milestone was achieved, the DC-8 was cleared for departure to Australia. A rehearsal flight was conducted on the night of June 8 prior to departure for Melbourne to practice instrument set-up and take-down, and to allow calibration using astronomical sources such as Venus. The final TCM-4, was executed during transit of the DC-8 to Melbourne on June 9th. Upon arrival in Melbourne, an orbital dynamics (OD) solution was supplied by the Deep Space Network (DSN) team at Jet Propulsion Laboratory (JPL) which provided the entry state information (at $\sim 200 \mathrm{~km}$ altitude). The OD solution was utilized for simulating the trajectory through the atmosphere from which position and timing information was used for planning the rehearsal flight track $24 \mathrm{~h}$ prior to re-entry (Figure 2). Continual tracking by the DSN team at JPL provided higher fidelity OD solutions, which were analyzed the morning of the re-entry to slightly refine the observation flight track. Just prior to departure for the observation mission ( $\sim 2 \mathrm{~h}$ prior to entry), confirmation of SRC release from the spacecraft bus was received. The SRC was released $\sim 3 \mathrm{~h}$ prior to entry with a predicted separation velocity of $0.17 \mathrm{~m} / \mathrm{s}$.

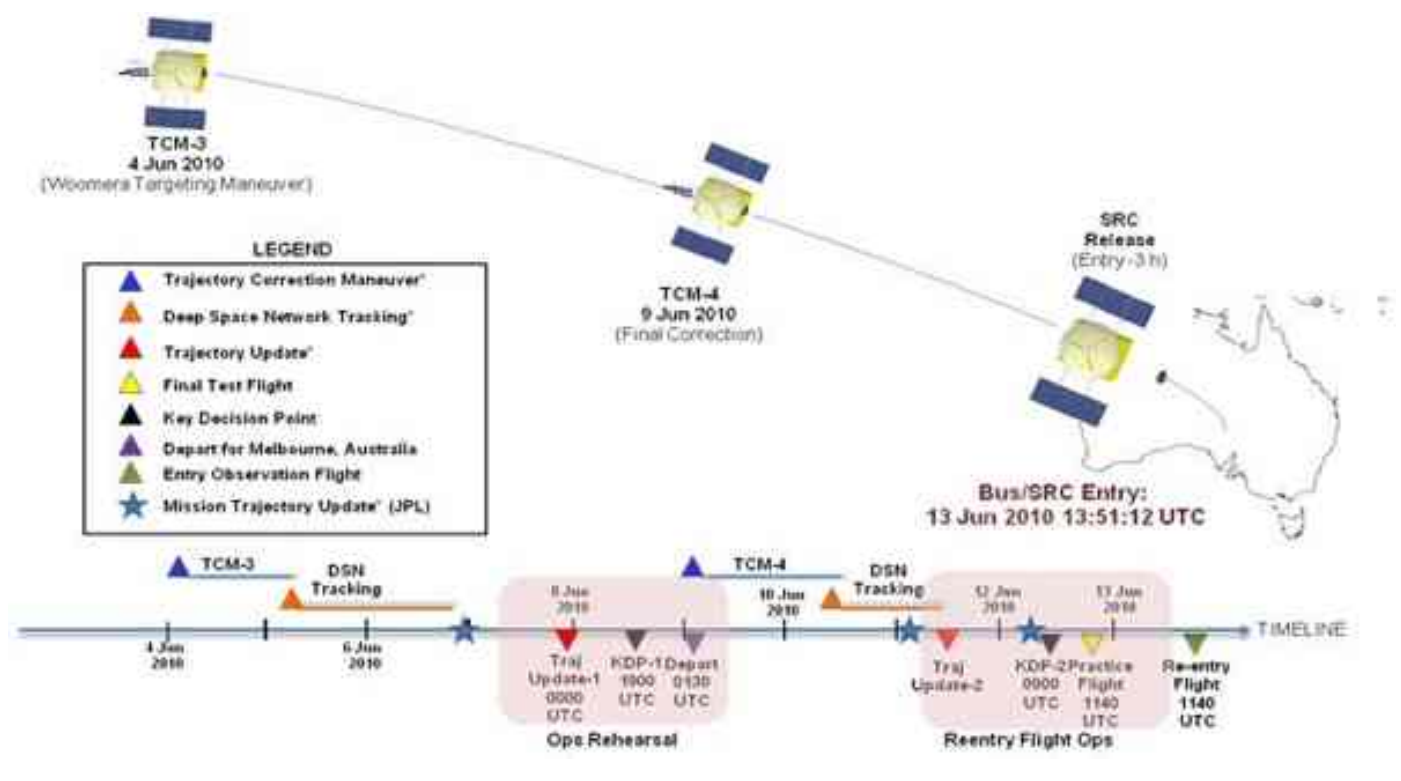

Figure 1- Hayabusa terminal approach event timeline shown with observation mission operational protocols.

A detailed overview of the Hayabusa Airborne Observation can be found in Ref. 6. A number of objectives and constraints had to be met in designing the observation flight track. Table 1 lists the primary objectives and constraints together with the rationale. Given the desire to observe as much of the re-entry as possible $(\sim 100-35 \mathrm{~km}$ altitude) and to collect the maximum amount of radiation with the scientific instruments on-board the DC- 8 , the flight track was designed to view the SRC nearly head-on while maintaining a range of $<250 \mathrm{~km}$ during the peak heating window. Furthermore, since the SRC is a blunted, 45 deg sphere cone, the velocity vector to observer angle was kept below 45 deg during the peak heating window so that the entire front surface area was observed as long as possible through peaking heating. At the same time, we had the contradicting requirement to provide a high view angle to be able to separate the bus from the capsule (separation distance of $\sim 2 \mathrm{~km}$ ). Due to safety concerns from potential spacecraft bus break-up debris, the aircraft was to maintain $+/-60 \mathrm{~km}$ keep out zone on either side of the entry trajectory. ${ }^{5}$ Finally, the DC-8 was to maintain level flight at $\sim 12 \mathrm{~km}$, with an elevation angle to the SRC below 16 deg to facilitate manual tracking of the SRC through the DC-8 windows. 


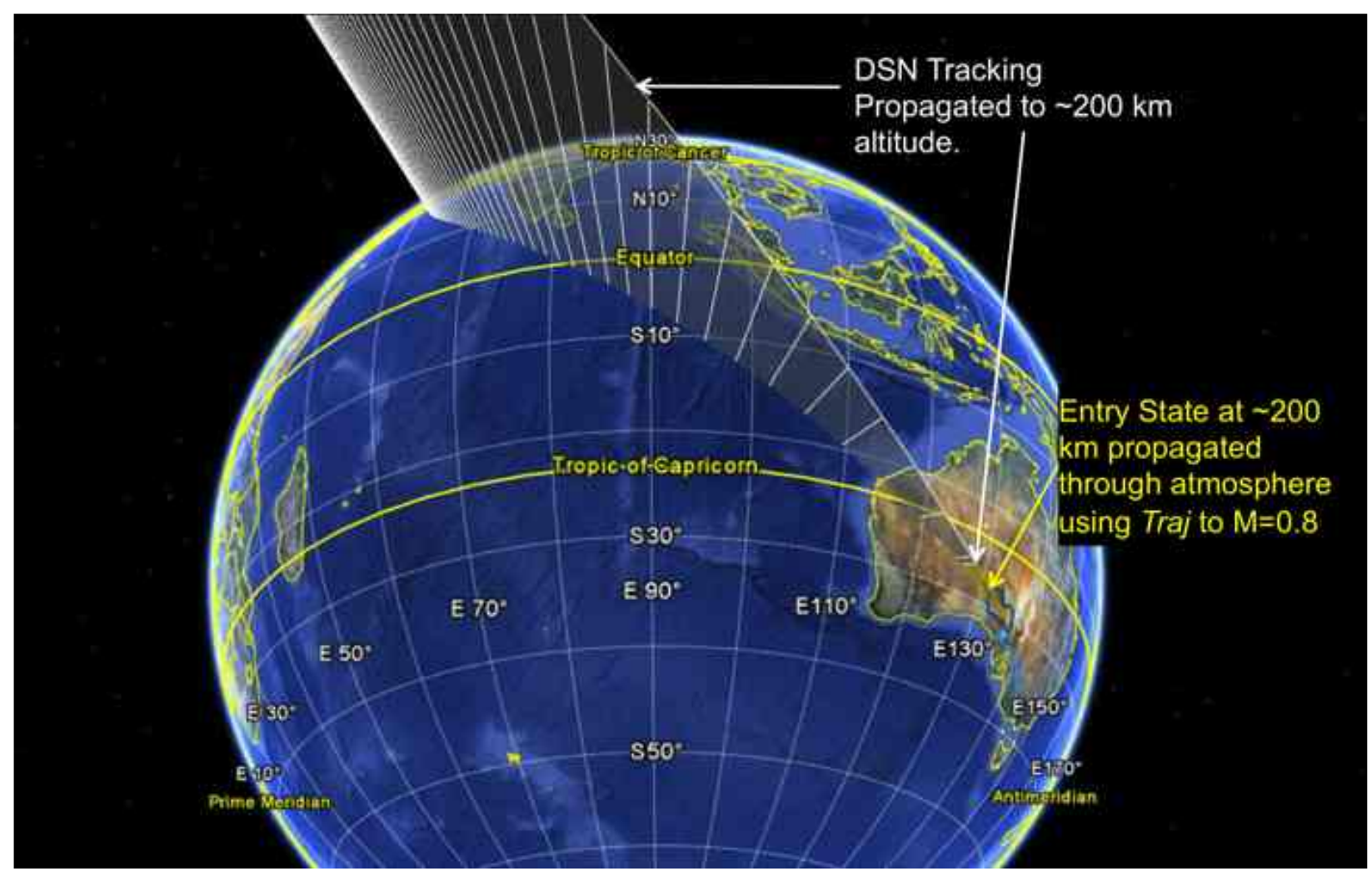

Figure 2- Hayabusa trajectory overview. Position tracking and navigation updates were provided by DSN. Entry states at $\sim 200$ $\mathrm{km}$ were used to simulate trajectories for observation mission planning.

\section{Observation Mission Design}

A suite of tools was used for observation mission design. Predicted trajectory and aerothermal heating environments were generated using TRAJ. ${ }^{7}$ An Excel/Visual Basic tool was utilized to design and trade observation flight tracks against the various observation mission design parameters from the predicted SRC trajectory. The outputs of these tools were then visualized within Satellite Took Kit (STK) to cross compare and calculate SRC timing, position and viewing geometries from the various observation points and to provide rapid graphical updates in two and three dimensions or as real-time animations. The peak heating point was the key event used in observation flight planning in order to determine the DC-8 position needed to achieve a boresight view (normal to the port side windows) at peak heating. The predicted peak heating point and position was input into the Excel/Visual Basic tool in order to analyze observation flight path options. The flight planning tool provides the location and timing data for two waypoints for the planned observation leg flight track. The waypoints were then given to the DC-8 navigator as inputs for the DC-8 navigation system.

\section{A. Trajectory and Entry Environments}

Predicted entry states were used to calculate the Hayabusa SRC trajectories from $\sim 200 \mathrm{~km}$ to main parachute deployment $(\mathrm{M}=0.8)$. Entry state updates were provided after each TCM was completed. Table 2 shows a set of inputs used for calculating the trajectory and aerothermodynamic environments using tracking data from the night prior to entry (OD110611_v2). Table 2 lists the Hayabusa Spacecraft Bus entry state information OD100611_v2 (tracking data to 05:00 UTC 12 Jun 2010). Table 2 also lists parameters from the last OD update (tracking data to 13:13 UTC, 13 Jun 2010) that was received after the entry had occurred. Figure 3 shows the total, convective and radiative heat flux as a function of altitude. The capsule was expected to become visible (depending upon camera sensitivity) around $100 \mathrm{~km}$ altitude, and peak heating was expected to occur near $57 \mathrm{~km}$ altitude. Tracking of the SRC was possible until $30 \mathrm{~km}$ at which point radiative emission could no longer be detected. 


\begin{tabular}{|c|c|c|}
\hline Parameter & $\begin{array}{c}\text { OD 100611_v2 } \\
\text { (Used for Flight Planning) }\end{array}$ & $\begin{array}{c}\text { Final Tracking Update } \\
\text { (13:13 UTC) }\end{array}$ \\
\hline Time (UTC) & $13: 51: 11.47$ & $13: 51: 12.00$ \\
\hline Altitude & $201.9223 \mathrm{~km}$ & $202.0308 \mathrm{~km}$ \\
\hline Latitude (geodetic) & -27.3614 & -27.3606 \\
\hline Longitude & 126.3783 & 126.3857 \\
\hline Velocity (inertial) & $12.0350 \mathrm{~km} / \mathrm{s}$ & $12.0348 \mathrm{~km} / \mathrm{s}$ \\
\hline Flight Path Angle (inertial) & $-12.3545 \mathrm{deg}$ & $-12.3501 \mathrm{deg}$ \\
\hline Heading (inertial) & $112.0557 \mathrm{deg}$ & $112.0676 \mathrm{deg}$ \\
\hline
\end{tabular}

Table 2- Entry state parameter inputs used for trajectory simulations prior to observation flight and for post flight analysis.

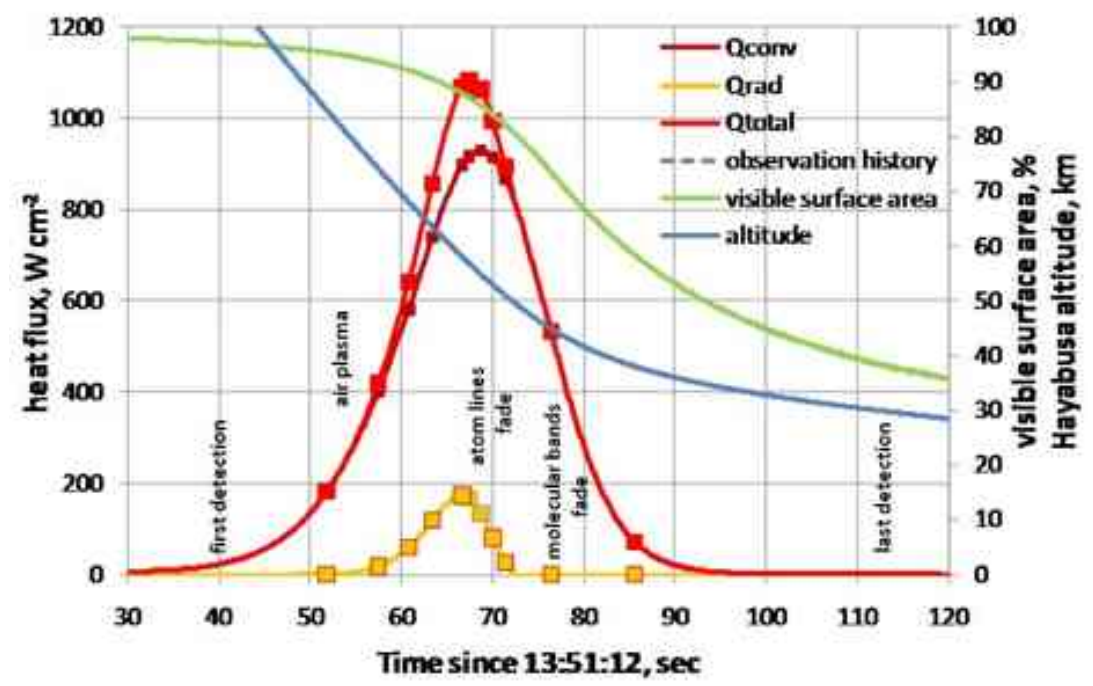

Figure 3- Calculated stagnation point aerothermodynamic environments from OD100611_v2. Also plotted is the nominal visible surface area as viewed from the nominal position of the DC-8.

\section{B. Flight Path Planning}

During the mission planning phase, predicted trajectory information was utilized to trade 30 candidate observation flight paths. The Excel/Visual Basic tool had previously been developed to analyze the effects of level flight and turning aircraft trajectories for the observation of the ATV re-entry. ${ }^{5}$ Parameters such as instrument pointing angles, range to target, angle between sight vector and SRC velocity vector, and elevation angle of target, reveal usually conflicting requirements that are easily traded within the tool. The observation flight path analysis outputs time, position and flight azimuth to be used by the aircraft pilots and navigator. Two baseline observation flight tracks on the North and South side of the entry trajectory were selected from the observation flight path trade study (Figure 4). Further refinements to these observation tracks were made upon completion of the various spacecraft TCMs and cross-checked to ensure all objectives and constraints had been met. One lesson learned from the ATV re-entry observation, compensation for winds aloft, was taken into account for the Hayabusa observation. The two practice observing flight legs, flown to synchronize arrival time to the first waypoint for the actual re-entry, were used to compensate for winds aloft in real-time so that instrument pointing would not be off nominal. Thus, an additional Excel tool was developed that would incorporate predicted/actual winds aloft and provide waypoints that the aircraft navigator could enter directly into the navigation system. The wind compensation tool takes time, position and flight azimuth for a key location 


\section{Longitude}

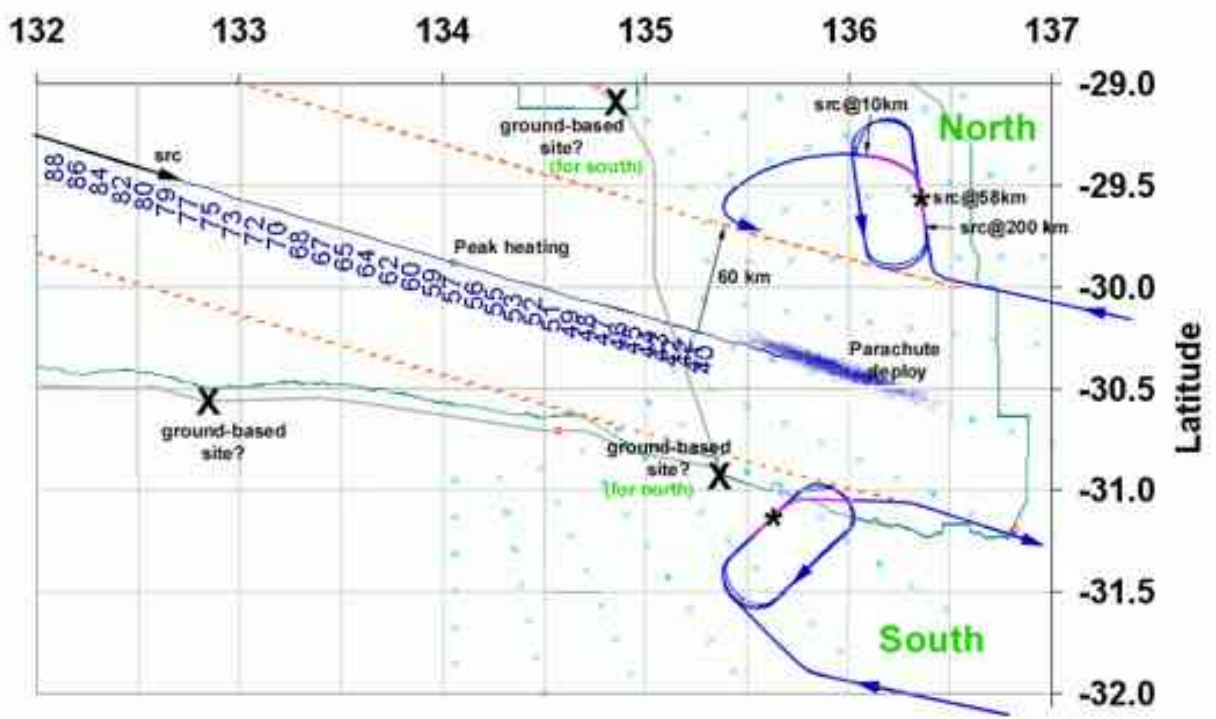

Figure 4- Initial south (primary) and north (back-up) observation flight tracks identified from trade study.

and compensates for wind velocity and direction. The tool can also project aircraft position forward or backward in time from the key location point. While wind velocity was not very high during the Hayabusa observation mission, the tool made compensating for wind and generation of the desired aircraft position and heading much easier. Pre and post observation, STK allowed us to confirm the accuracy and timing of the predicted SRC entry trajectory with the planned and as flown DC-8 flight tracks. Azimuth, elevation and range data was computed for the various observation locations, namely the DC-8 and locations of the ground based observers at Coober Pedy, Tarcoola West and Kingoonya. A comparison between elevation and range data for the predicted SRC trajectory and the observer locations is shown in Figure 5.

\section{Preparation for Entry Observation}

Except for a few fixed widefield cameras, all instruments

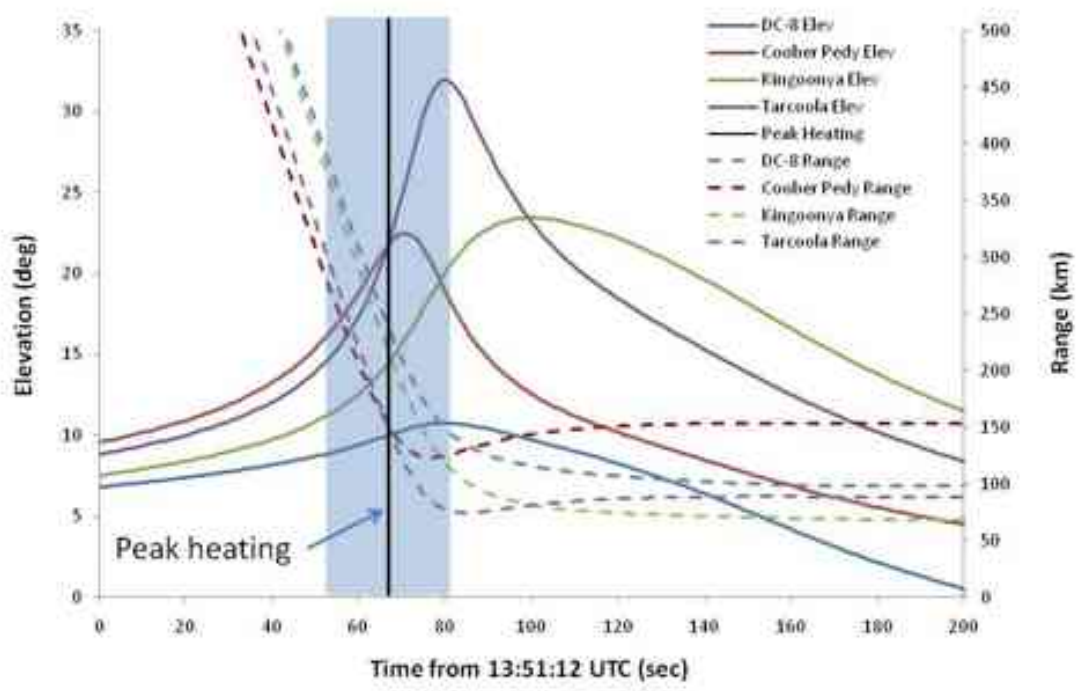

Figure 5- Elevation and range data calculated from the DC-8 and ground-based observation positions at Coober Pedy, Kingoonya and Tarcoola West. 
manually tracked the spacecraft following acquisition. In order to maximize the amount of data collected it was important that instrument operators became familiar with the star-field that the SRC was to traverse. Since the Stardust re-entry observation mission, the team has used Skymap, a shareware program which was originally developed for the amateur satellite observing community. ${ }^{8}$ It supports both Two Line Elements (TLEs) for orbiting objects, and position (time, latitude, longitude, altitude) files for arbitrary trajectories. It also supports observer position files with labels for each of the positions. Figure 6 shows an example of the trajectory overlaid with the star background as viewed from the DC-8. The SRC and spacecraft bus were expected to become visible between the constellations Leo and Coma Berenices. As the SRC traversed the sky, the track would take it through the bottom portion of the constellation Coma Berenices. Peak heating was expected to occur at $\sim 57 \mathrm{~km}$, whereby the SRC would have just left the constellation Coma Berenices. As viewed from the NASA DC-8, the SRC was never higher than 11 deg elevation.

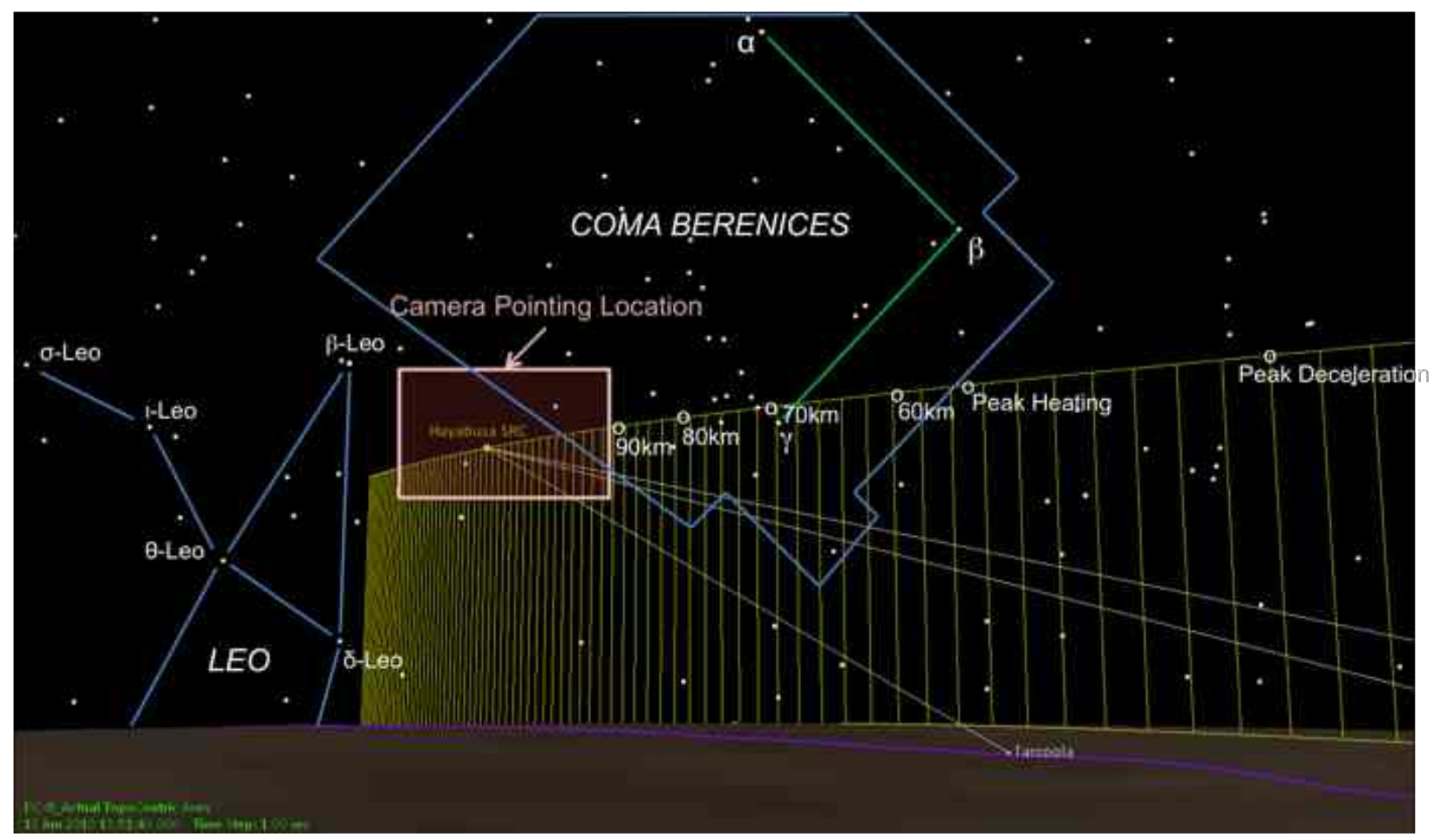

Figure 6- Simulated view of predicted trajectory (13 Jun 2010 13:51:43 UTC) overlaid on star-field background as viewed from the DC-8. Magnitude +6 or brighter stars shown for clarity.

A key event timing script was also prepared and called out over the DC-8 intercom system. The script provided timing cues for SRC tracking by the instrument operators. The events of the script were called out during rehearsal and observation. Table 3 lists the script events and times. Once the DC- 8 maintained level flight along the observation leg, "wings level" was announced. This was followed by a series of announcements to prepare the science team for camera pointing. The capsule was expected to be bright enough for tracking just prior to the "In Coma Berenices" event, where the operators were instructed to have their cameras pointed against the star background (see Figure 6). The remaining key event cues were to aid in tracking in case off-nominal pointing of the instrument occurred.

\begin{tabular}{|c|c|}
\hline Time (UTC) & Event \\
\hline $13: 50: X X$ & "Wings Level" \\
\hline $13: 50: 42$ & "30 seconds to Interface" \\
\hline $13: 51: 12$ & "200 km" \\
\hline $13: 51: 47$ & "100 km" \\
\hline $13: 51: 59$ & "In Coma Berenices" \\
\hline $13: 52: 05$ & "Bus Break-Up" \\
\hline
\end{tabular}




\begin{tabular}{|c|c|}
\hline $13: 52: 19$ & "Peak Heating" \\
\hline $13: 52: 26$ & "Peak Deceleration" \\
\hline $13: 52: 34$ & "40 km" \\
\hline $13: 52: 44$ & "35 km" \\
\hline
\end{tabular}

Table 3- Key event cues used for SRC tracking. 


\section{Observation Mission Execution}

The observation mission was based out of Melbourne's international airport. Approximately $12 \mathrm{~h}$ prior to entry, the observation flight path was filed with the Australian authorities. Figure 7 shows an overview of the observation mission. The DC-8 departed Melbourne International Airport approximately $2 \mathrm{~h}$ prior to entry, heading northwest towards the observation staging location just south of the WPA. During transit to the staging location, the instruments were set-up and final check-outs performed. Approximately 35 min prior to entry, the DC-8 was maneuvered into position to execute the race track patterns for two practice legs and the observation leg. The difficult task of manual SRC tracking during the observation period was made easier through constant updates to the preflight prediction of the capsule's trajectory and execution of the practice legs prior to entry. Figure 8 shows the actual position of the DC-8, taken from GPS data. Two waypoints were provided to the DC-8 navigator (M.E.A.) in order to position the DC-8 at the proper orientation needed to meet the viewing objectives. During practice legs \#1 and \#2, the DC-8 was within $+/-1 \mathrm{sec}$ of the planned positions of Waypoints \#1 and \#2. Nominally the DC-8 was flown at an altitude of $12.5 \mathrm{~km}$ at an average airspeed of $811 \mathrm{~km} / \mathrm{h}(504 \mathrm{miles} / \mathrm{h})$ on the observation leg.

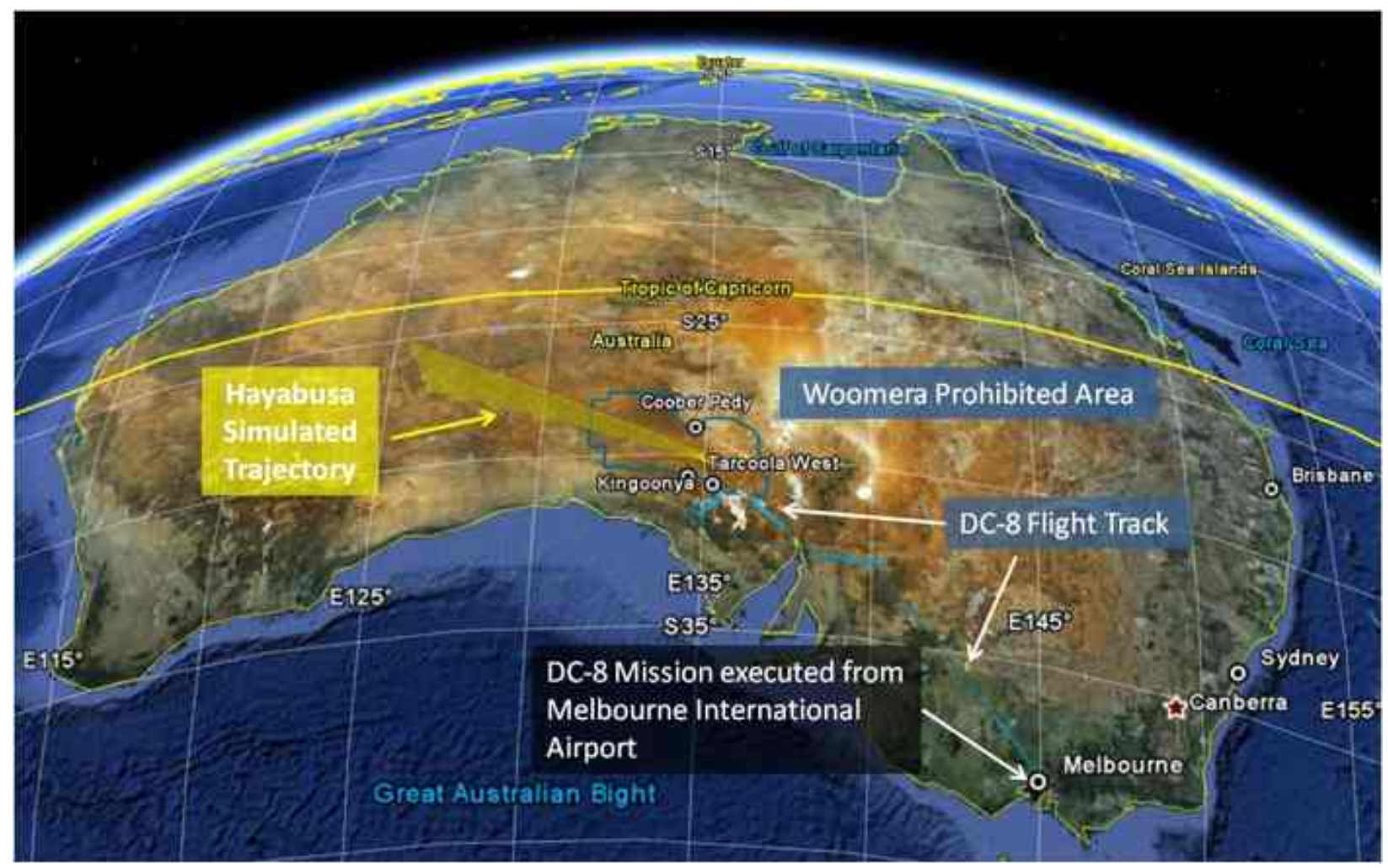

Figure 7- Overview of the Hayabusa Airborne Observation mission.

Although there was great uncertainty regarding whether or not the SRC would be discernible from the break-up of the spacecraft bus, the DC-8 positioning and timing were nearly flawless. During the planned observation leg track, waypoint \#1 was achieved on time, and Waypoint \#2 was $\sim 6$ seconds late. Even with arriving slightly late at waypoint \#2, the SRC was nearly boresight at peak heating. Figure 3 shows the percentage of visible surface area calculated from actual GPS data and the simulated SRC trajectory. All planned observation mission objectives were met. Careful coordination between the observation mission planning team and the DC- 8 navigator enabled the science team to image the entry event and obtain valuable spectroscopic data on both the SRC and the break-up of the spacecraft bus ( 23 out 25 instruments were successful). 


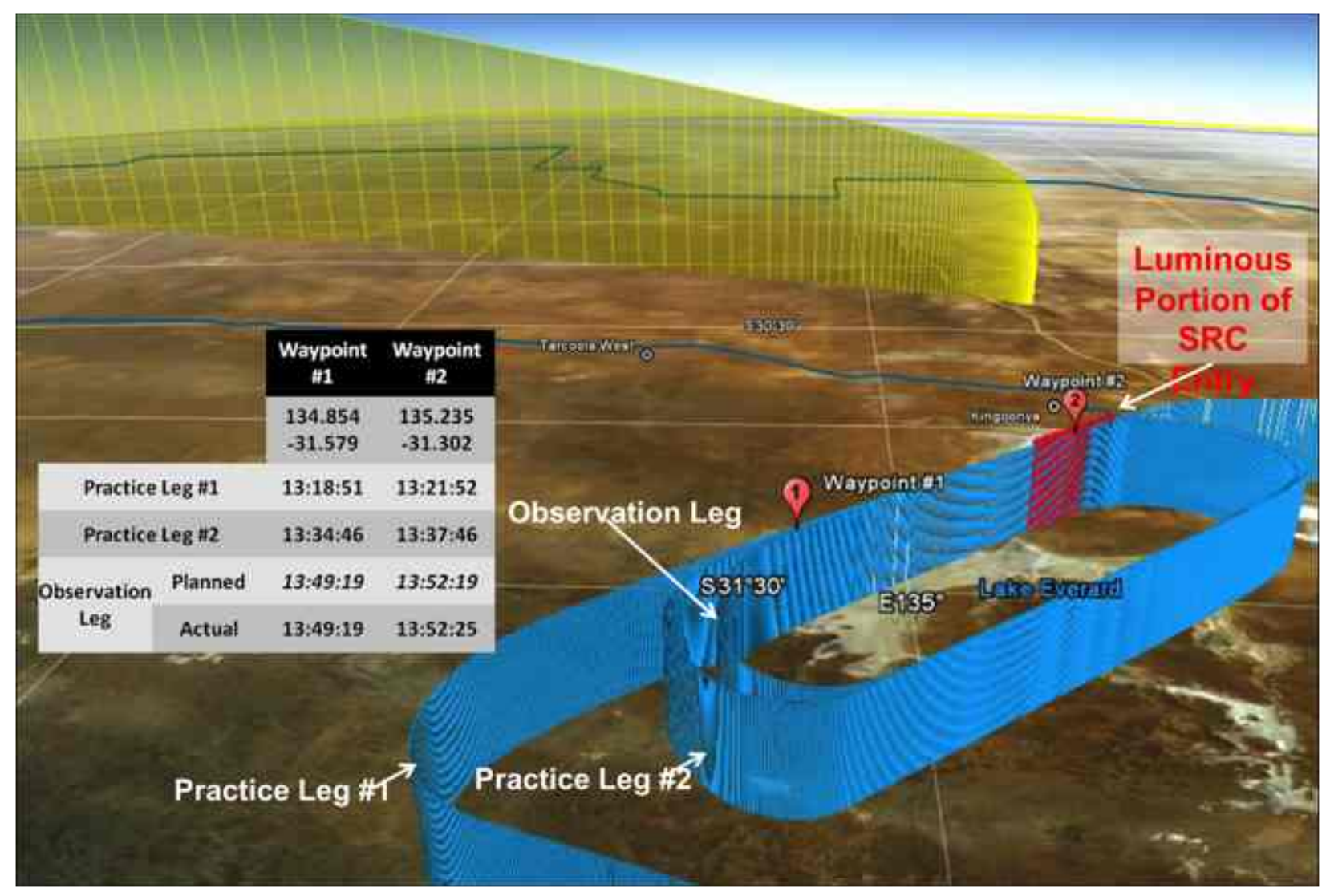

Figure 8- Close-up view of the as-flown observation mission flight track, displaying the two waypoints used for aircraft navigation and the racetrack pattern flown (two practice legs and observation leg).

\section{Conclusions}

The Hayabusa re-entry observation mission enabled spectroscopic instruments on board the NASA DC-8 to view the incoming SRC and break-up of the spacecraft bus. A combination of trajectory analysis, observation flight planning tools, scenario modeling and systems engineering processes were utilized for the observation mission design. Based upon the objectives and constraints, a primary (South of WPA) and alternate (North of SRC ground track) observation flight track were designed from key event timing and position along the SRC trajectory. To aid in SRC tracking, star charts overlaid with the SRC trajectory were provided to the instrument operators and ground-based observers. The as-flown observation track was successfully executed and achieved all mission objectives. 23 out of 25 scientific instruments onboard the DC-8 returned useful data of the SRC re-entry and spacecraft bus break-up. Mission success was realized through careful planning and coordination with the various mission stakeholders, including representatives from NASA, JAXA and Australia.

\section{Acknowledgments}

The authors would like to gratefully acknowledge the NASA DC-8 crew and the personnel supporting operations at the Dryden Aircraft Operations Facility. Special thanks to L. Huynh, D. Prabhu, D. Kontinos, D. Jordan at NASA ARC, T. Oram of NASA JSC, T. Thompson, R. Haw of NASA JPL, I. Tuohy (BAE Systems), J. McKevett (AOSG), T. Yamada and H. Tanno of JAXA is also appreciated.

\section{References}

\footnotetext{
${ }^{1}$ Kuninaka, H., Kawaguchi, J. "Lessons Learned from the Round Trip of Hayabusa Asteroid Explorer in Deep Space” IEEE Aerospace Conference, Paper \#1771, 2011.
} 
${ }^{2}$ Kawaguchi, J., Mukai, T., Abe, M., Yoshikawa, M. "Curation and Initial Analysis Readiness for Sample Returned by Hayabusa” 38th COSPAR Scientific Assembly, July 15-18, 2010, Bremen, Germany.

${ }^{3}$ Jenniskens, P., Wercinski, P.F., Olejniczak, J., Wright, M., Raiche, G., Kontinos, D., Desai, P., Spalding, R.E., Sandquist, K., Rossano, G., Russell, R.W., Revelle, D.O., Hladiuk, D., Hildebrand, A.R. "Surface Heating from Remote Sensing of the Hypervelocity Entry of the NASA GENESIS Sample Return Capsule" $44^{\text {th }}$ AIAA Aerospace Sciences Meeting and Exhibit, Jan 9-12, 2006, Reno, Nevada.

4 "Stardust Hypervelocity Entry Observing Campaign Support" NASA Engineering and Safety Center Report, RP-06-80, August 31, 2006.

${ }_{5}^{5}$ De Pasquale, E., Francillout, L., Wasbauer, J.-J., Hatton, J., Albers, J., Steele, D., “ATV Jules Verne Re-entry Observation: Mission Design and Trajectory Analysis" 30 ${ }^{\text {th }}$ IEEE Aerospace Conference, Paper \#1155, Mar. 2009.

${ }^{6}$ Grinstead, J. H., Jenniskens, P., Cassell, A.M., Albers, J., Winter, M. W. “Airborne Observation of the Hayabusa Sample Return Capsule", paper \#- AIAA $42^{\text {nd }}$ Thermophysics and Heat Transfer Conference, 27-30 Jun, 2011, Honolulu, HI, USA.

7 Allen, G. A., Jr., Wright, M. J., and Gage, P., "The Trajectory Program (Traj): Reference Manual and User's Guide", NASA TM -2005-212847, 2005.

${ }^{8}$ Matson, R. Skymap v 6.5, http://www.vms.utexas.edu/ ecannon/sky65.zip 